\title{
Novel approaches to target the antibiotic resistance in short term
}

\begin{abstract}
Antibiotic resistance is a big global threat across the world due to rapid emergence of multidrug resistant bacterial strains. Scientists, healthcare professionals and pharmaceutical industry are significantly facing this problem. Gram negative bacteria (carbapenemresistant Enterobacteriaceae) are considered the most critical priority in the list of high risk to antibiotic resistance by WHO. It is difficult to develop the new antibiotics promptly against the rapidly growing multidrug resistant strains. This mini review describes the various innovative ways to combat the antibiotic resistance in short time.
\end{abstract}

Keywords: antibiotic resistance, enterobacteriaceae, loperamide, pseudomonas aerogenosa, acinetobacter baumannii
Volume 4 Issue 6 - 2017

\author{
Anju Kaushal \\ Experienced Microbiologist/Biotechnologist, Panjab University, \\ India
}

Correspondence: Anju Kaushal, Experienced Microbiologist/ Biotechnologist, Panjab University, Chandigarh, India, Tel 9878743795,Email anjukaushal/86@yahoo.com

Received: May 15, 2017 | Published: June 16, 2017

\section{Summary}

One of the biggest threats to global health today is antibiotic resistance. There is a big concern that what is being done to combat this. Because bacteria develop antibiotic resistance faster as compared to the new development of antibiotics which takes years in its development.

Antibiotic resistance is a challenge in the treatment of diseases today, as bacteria continuously mutate and develop resistance against multiple drugs design to kill them, turning them in to superbugs. Most commonly Gram negative bacteria gain the resistance to multiple antibiotics, no new drug will be available to treat these infections in near future e.g. carbapenem- resistant Enterobacteriaceae. WHO revealed that gram negative bacteria are frequently resistant to antibiotics in the 12 families of bacteria. The main contributing factor to increased drug resistance is permeability barrier caused by the outer membranes of bacteria.

Synergistic use of antibiotics with FDA approved non-antibiotics is a novel approach which enable to breach the outer membrane barrier. In this innovative strategy non-antibiotic compounds boost the effectiveness of drugs. Anti-diarrheal medication loperamide enhance the effectiveness of eight different antibiotics. Minocycline with tetracycline along with drug benserazide increases the antibiotic activity against Pseudomonas aerogenosa, a causative agent of hospital acquired infections. Polymixin is known as effective drug against $K$. pneumonae, P. aerogenosa and Acinetobacter baumannii, plasmid transferable gene mcr-1 was discovered to create resistance to polymixin-E in 2015.Different combinations have been tried to increase the effectiveness. Without new antibiotics in near future, we must explore innovative approaches to preserve the clinical utility of important last-line antibiotics such as the polymyxins. ${ }^{1,2}$ Commented by Professor Jian Li, Head of the Laboratory of Antimicrobial Systems Pharmacology from Monash University, Victoria, Australia.

The beneficial effects of ivacaftor and lumacaftor combined with polymixin $\mathrm{B}$ increase the effectiveness to treat cystic fibrosis and closantel used to treat the parasitic worm infection. According to Schneider and co-authors, berberine (plant extract from barberry) with ampicillin or oxacillin are effective against MRSA strains. Scientists of US and India successfully modified the precursor of rifampicin, a new compound 24-desmethylrifampicin has potency against the multidrug resistant strains of tuberculosis bacteria. The McMaster scientists have found an anti-convulsant drug called lamotrigine is the first chemical inhibitor of the assembly of ribosomes in bacteria. Ribosomes are the molecular machines in cells that create all proteins. Many antibiotics attack what ribosomes do. However, the McMaster team found that lamotrigine stopped ribosomes from being created in the first place.

A team of five researchers and clinicians in Singapore led by Dr Desmond Heng, ICES Institute of Chemical and Engineering Sciences and NUH National University Hospital have discovered new ways to use a combination of four new drugs formulations that are each made up of three different antibiotics to fight against the respiratory system infections and antibiotic resistant superbugs. These drugs are effective against Pseudomonas aerogenosa and Klebsiella pneumoniae. These formulations kill bacteria five times more effectively than antibiotics used for treating respiratory system infection these days.

Researchers of Rockefeller University and their collaborators are working for smarter antibiotics. A programmable antibiotic technique selectively targets the bacteria which harbor the resistant strains while leaving other microbes alone, which wipe out the bad bugs at once. ${ }^{3}$ They succeeded by instructing a bacterial enzyme "Cas9" to target the particular DNA sequence and cut it up. This approach leaves the microbial community intact and can keep the resistance in check and prevent certain type of secondary infections, eliminating the two hazard associated with treatment by classical antibiotics.

In the development of rapid test kits showing what bacterial infection and what antibiotics they are resistant to will have huge impact on un-necessary use of antibiotic, hence reduce their excessive use. Even the modified version of vancomycin increases the potency and reduces the side effects. Rediscovery of old antibiotics like octapeptins are now being developed to combat Gram-negative "superbugs". ${ }^{4}$ Repurposing drugs like fexinadole which had some success in 1970s, have been identified for treatment of sleeping sickness is undergoing phase III trial. Novobiotic pharmaceuticals discovered teixobactin (new antibiotic) by in situ isolation of soil bacteria by using "ichip".

Last approach is the community for Open Antimicrobial Drug Discovery is crowd sourcing the new antibiotics by searching on new compounds sourced from academic chemists around the world. Dr 
Derry Mercer, principal scientist at NovaBiotics Ltd, said "Research and development of new anti-microbials remains a vitally important pursuit for combating the problems of antibiotic resistance, but alternative approaches to this problem are also urgently needed".

Non-antibiotic strategies like probiotic therapies or vaccines can help to reduce the excessive use of antibiotics. Antibiotics are never replaced by these but these can assist in preserving the effectiveness of the antibiotic drugs.

It is hard to predict which approach is best to combat antibiotic resistance in short time. However, it is important that all the possible avenues should be considered. Methods also include as vaccine development, bacteriophage therapy, targeting biofilms should also be urgently examined.

\section{Acknowledgements}

None.

\section{Conflict of interest}

The author declares no conflict of interest.

\section{References}

1. Elena K Schneider, Felisa Reyes-Ortega, Tony V, et al. Antibiotic-nonantibiotic combinations for combating extremely drug-resistant Gramnegative 'superbugs'. Essays In Biochemistry. 2017;61(1):115-125.

2. Jonathan MS, Joseph HD, Chand SM, et al. Discovery of a small molecule that inhibits bacterial ribosome biogenesis. ELife. 2014;3:e03574.

3. Nigam A, Almabruk KH, Saxena A, et al. Modification of Rifamycin Polyketide Backbone Leads to Improved Drug Activity Against Rifampicin-Resistant Mycobacterium tuberculosis. J Biol Chem. 2014;289(30):21142-52.

4. Bikard D, Euler CW, Jiang W, et al. Exploiting CRISPR-Cas nucleases to produce sequence-specific antimicrobials. Nat Biotechnol. 2014;32(11):1146-1150 